\title{
QUALITY IMPROVEMENT Use of DECAF scoring system to facilitate early discharge in acute exacerbation of COPD patients: a quality improvement project at a district general hospital
}

\author{
Authors: Iftikhar Nadeem, ${ }^{\mathrm{A}}$ Alexander Light, ${ }^{\mathrm{B}}$ Callum Donaldson, ${ }^{\mathrm{B}}$ Usman Feroze Khatana, ${ }^{\mathrm{C}}$ Dinesh Bagmane, ${ }^{\mathrm{D}}$ \\ Enson Thomas ${ }^{\mathrm{D}}$ and Mohammed Azher ${ }^{\mathrm{D}}$
}

\section{Introduction}

DECAF is a scoring tool that can predict severity in patients with an acute exacerbation of chronic obstructive pulmonary disease (AECOPD). Previous research has shown AECOPD patients with DECAF scores of $0-1$ are candidates for early discharge.

\section{Methods}

Plan, do, study, act (PDSA) methodology was used. Patients with AECOPD and a DECAF score of 0-1 were included. Notes were retrospectively reviewed for patients for DECAF score, length of stay, 30-day re-admission and 30-day mortality (PDSA cycle 1). A framework to facilitate early discharge for patients was subsequently established. Awareness was increased through teaching sessions, posters and targeted emails. To evaluate our improvements, the same parameters were collected prospectively (PDSA cycle 2 ).

\section{Results}

DECAF score was assessed for no patients in PDSA cycle 1 $(n=20)$ but was assessed for all patients in PDSA cycle 2 $(n=14)$. Hospital stay was significantly decreased in PDSA cycle 2 (mean $0.29 \pm 0.45$ days) compared with PDSA cycle 1 (mean $3.71 \pm 2.69$ days; difference $p<0.00001$ ). Thirty-day re-admission and 30-day mortality was not significantly different between two groups.

Conclusion

DECAF protocol is safe and feasible in the district general hospital setting and can facilitate early discharge for patients with low severity AECOPD without any worrisome effects.

KEYWORDS: DECAF, COPD, early discharge

DOI: 10.7861/fhj.2020-0097

Authors: ${ }^{\text {A }}$ respiratory specialty trainee-5, Lister Hospital, Stevenage, UK; ${ }^{\text {B }}$ foundation year-2 doctor, Addenbrookes Hospital, Cambridge, UK; ${ }^{C}$ respiratory specialty trainee-3, Peterborough Hospital, Peterborough, UK; ${ }^{D}$ respiratory consultant, Bedford Hospital, Bedford, UK

\section{Introduction}

Acute exacerbation of chronic obstructive pulmonary disease (AECOPD) is a common cause of hospital admission, morbidity and mortality. In England, COPD causes 115,000 emergency admissions per year. ${ }^{1}$ According to the 2017 UK COPD clinical audit, mortality for COPD patients stands at $6.1 \%$ at 30 days and $11.3 \%$ at 90 days, with readmission rates of $24.8 \%$ and $43.1 \%$ within 30 and 90 days, respectively. ${ }^{2}$

In 2012, the DECAF prognostication tool was developed in an English population to risk-stratify these patients. ${ }^{3}$ Scored out of 6 , the five parameters used are dyspnoea, eosinopenia, consolidation, acidaemia and atrial fibrillation (Table 1).

In both internal and external validations, DECAF demonstrated excellent discrimination for predicting inpatient mortality, and was superior to other prognostic scores. ${ }^{4}$ The DECAF score is advantageous in being easy to calculate with standard parameters at the patient's bedside, and providing the clinician a quantifiable risk assessment method for an AECOPD patient. In a randomised controlled trial, facilitating early discharge for low-risk patients (DECAF score $0-1$ ) instead of usual care was preferred by patients, was economically favourable and had similar rates of readmission and mortality. ${ }^{5}$ Thus, if used correctly, DECAF improves utilisation of hospital resources by reducing inappropriate admissions, but also helps flag up high-risk patients

Table 1. Parameters included in the DECAF score

$\begin{array}{ll}\text { Parameter } & \text { Score } \\ \text { Dyspnoea }(\mathrm{eMRCD} 5 \mathrm{a}) & 1 \\ \text { Dyspnoea }(\mathrm{eMRCD} 5 \mathrm{~b}) & 2 \\ \text { Eosinopenia }\left(<0.05 \times 10^{9} / \mathrm{L}\right) & 1 \\ \text { Consolidation } & 1 \\ \text { Acidaemia }(\mathrm{pH}<7.3) & 1 \\ \text { Atrial fibrillation (including known paroxysmal } & 1 \\ \text { atrial fibrillation) } & \\ \text { Total } & \mathbf{1 6}\end{array}$


in need of treatment escalation. In this quality improvement project (QIP), we aimed to implement a DECAF protocol in an English district general hospital (DGH) in order to facilitate early discharge for low-risk AECOPD patients.

\section{Methods}

\section{Overview}

Our study is reported in line with SQUIRE 2.0 guidelines. We designed and carried out our QIP with plan, do, study, act (PDSA) methodology. The QIP was registered with our local audit department though, given the nature of this project, no formal ethical approval was required.

\section{PDSA cycle 1}

Plan

This QIP took place at a busy, 400-bed DGH (Bedford Hospital) in the east of England. Our working group comprised multiple healthcare workers including consultants, junior doctors and respiratory clinical nurse specialists.

Our primary objective was to introduce a protocol for patients presenting to hospital with AECOPD to facilitate early discharge for appropriate candidates (DECAF score 0-1), particularly over the winter period. This protocol would include an option for patients to be discharged under a hospital-at-home (HAH) treatment plan. Patients with higher DECAF scores would be admitted and treated according to British Thoracic Society guidelines. ${ }^{6}$

\section{Do}

In November 2019, we performed a retrospective review of patients who were admitted to hospital with AECOPD between 1 September 2019 and 30 September 2019. These patients were identified through clinical coding of the diagnosis listed in their discharge summary.

Items for the following data fields were collected: days admitted, DECAF score including scores for individual parameters, and whether a DECAF score had been formally documented in either the patient's emergency department or acute medical clerkings. We also identified inpatient mortality and whether the patient died or was readmitted within 30 days of discharge with a repeat episode of AECOPD. Where an extended Medical Research Council Dyspnoea (eMRCD) score was not documented, we studied the patient's full medical and nursing notes and previous outpatient letters to approximate their score. If we were not able to obtain notes or adequate details from the notes to calculate DECAF for eligible patients, these patients were excluded from analyses.

\section{Study}

Following data collection, we calculated the number of patients in each of two DECAF score categories: 0-1 or higher. For the purpose of designing our protocol, we subsequently only included patients with a DECAF score of 0-1. Other exclusion criteria included patients admitted with an acute diagnosis other than, or in addition to, AECOPD and development of an acute clinical issue in addition to AECOPD during their inpatient stay. We also analysed mean length of stay for each category and compared values using the two-tailed Fisher's exact test.
Act

Following our study phase, our working group met to devise a new protocol that would be appropriate from both clinical and economic perspectives, and that was individually agreed upon by the respiratory department (including respiratory clinical nurse specialists) and acute medicine department (Fig 1).

To raise awareness of our initial findings and our new protocol, members of our working group presented our data at six regularlyheld teaching or audit sessions: respiratory departmental teaching, emergency department teaching, acute medicine departmental teaching, foundation year doctor teaching, core medical trainee teaching, and the hospital-wide audit and QIP meeting. We also produced posters highlighting our protocol and placed these in highly visible areas within both clinical and nonclinical areas of the emergency department, acute medical ward and respiratory wards.

\section{PDSA cycle 2}

\section{Plan}

Following local dissemination of our results and protocol, we planned to analyse the impact of our new protocol. We wished to study both variables: whether our protocol had been taken up by the relevant departments and whether this had affected clinical outcomes.

\section{Do}

We prospectively collected data on all patients presenting to the emergency department with AECOPD and a DECAF score of 0-1 from 15 February 2020 to 14 March 2020. Exclusion criteria were the same as for PDSA cycle 1. To identify eligible patients, the clerking notes of all medical patients who presented to the emergency department were reviewed by at least one member of our working group. The same data items as for our initial retrospective review were collected. Qualitatively, we also identified feedback of our protocol's strengths and limitations.

\section{Study}

We analysed the following outcomes: whether DECAF score was documented, mean length of stay, inpatient mortality, 30-day AECOPD-specific readmission rate and 30-day mortality.

Length of stay for DECAF 0-1 patients was compared between patients in our initial retrospective cohort and this prospective cohort using the two-tailed Mann-Whitney $U$ test. Thirty-day AECOPD-specific readmission rate, and 30-day mortality data were also compared between these two cohorts using the two-tailed Fisher's exact test. Statistical significance was assumed at $p<0.05$ in all analyses.

Act

To disseminate our findings, we re-presented our updated results at our hospital-wide audit and QIP meeting.

\section{Results}

\section{PDSA cycle 1}

During PDSA cycle 1, 31 patients attended our centre with AECOPD. Of these, 26 of these patients had notes available, with 20 patients meeting eligibility criteria. DECAF score was 
All patients attending hospital with an acute exacerbation of COPD should be DECAF scored

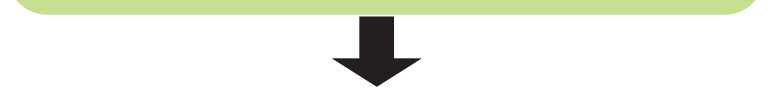

\begin{tabular}{|c|c|}
\hline Dyspnoea - & $\begin{array}{l}\text { 0: not too dyspnoeic to leave the house } \\
\text { 1: too dyspnoeic to leave the house but } \\
\text { independent with washing and dressing } \\
\text { 2: too dyspnoeic to leave the house and } \\
\text { wash or dress independently }\end{array}$ \\
\hline Eosinopenia - & $\begin{array}{l}0: \text { eosinophils }>0.05 \times 10^{9} / \mathrm{L} \\
\text { 1: eosinophils }<0.05 \times 10^{9} / \mathrm{L}\end{array}$ \\
\hline Consolidation - & $\begin{array}{l}0 \text { : No consolidation on chest X-ray } \\
1 \text { : consolidation on chest } \mathrm{X} \text {-ray }\end{array}$ \\
\hline Acidaemia - & $\begin{array}{l}0: p H>7.3 \text { on } A B G \\
1: p H<7.3 \text { on } A B G\end{array}$ \\
\hline Fibrillation (atrial) - & $\begin{array}{l}0: \text { no current or past history of } A F \\
1: \text { current or past history of } A F\end{array}$ \\
\hline
\end{tabular}

Patients with a total score of $0-1$ are candidates for early discharge.

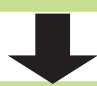

To facilitate discharge.

- Respiratory nurses can be contacted on bleep 566 to provide same day home oxygen and community follow-up if needed - If the attending doctor has ongoing concerns surrounding discharge, the respiratory registrar can be contacted on bleep 175 and, if unavailable, contact the respiratory consultant on call through the switchboard to take responsibility of the discharge.

Fig 1. Early discharge for chronic obstructive pulmonary disease exacerbation. $A B G$ = arterial blood gases; $A F=$ atrial fibrillation; $\mathrm{COPD}=$ chronic obstructive pulmonary disease.

assessed for zero patients. Mean length of stay in hospital was 3.71 days. Subgroup analysis revealed that length of stay was not significantly different between patients with DECAF scores 0 (mean 3.95 \pm 2.89 days; $n=11$ ) and 1 (mean 3.41 2.37 days; $n=9$; difference $p=0.88$ ). Readmission with AECOPD within 30 days of discharge was observed in two patients (10\%). Mortality within 30 days of discharge did not occur for any patient.

\section{PDSA cycle 2}

During PDSA cycle 2, 29 patients attended our centre with AECOPD. All of these patients had notes available, with 14 patients meeting eligibility criteria. DECAF score was assessed and documented for all 14 patients. All of these patients were seen by the respiratory team and those with DECAF score 0-1 were deemed fit for discharge under an HAH treatment plan, provided they did not have any issues related to discharge planning. This was observed for four patients and they were admitted to the wards. As part of our protocol, all patients were called by a respiratory clinical nurse specialist the next day and a week after discharge to review their progress. No patient required a visit by a respiratory nurse specialist. Prior to this, there was no formal early discharge service operational at our hospital for AECOPD patients and discharge decision was primarily a clinician-led decision.

Mean length of stay was $0.29 \pm 0.45$ days. This was very significantly decreased compared with PDSA cycle 1 ( $p<0.00001)$. Subgroup analysis demonstrated that length of stay was not significantly different between patients with DECAF scores 0 (mean $0.17 \pm 0.37$ days; $n=6$ ) and 1 (mean $0.5 \pm 0.5$ days; $n=8$; difference $p=0.33$ ). No patient was readmitted with AECOPD within 30 days of discharge, which was a non-significant difference compared with PDSA cycle $1(p=0.50)$. No patient experienced mortality within 30 days of discharge, the same as for PDSA cycle 1.

\section{Discussion}

\section{Summary of results}

Following introduction of a DECAF protocol to facilitate early discharge for low-risk AECOPD patients, our results demonstrate a significant decrease in mean length of stay for these patients with no significant difference in 30-day readmission or mortality.

\section{DECAF in the literature}

In its original development study of 920 patients, Steer et al showed that mortality increased progressively with DECAF score. $^{3}$ Patients with DECAF scores of 0 and 1 were classified as low risk. Inpatient mortality was calculated at $0.5 \%$ and $2.1 \%$, respectively, while 30 -day mortality was calculated at $1.5 \%$ and $3.8 \%$, respectively. Of studied parameters, eMRCD $5 \mathrm{~b}$ (odds ratio 7.30 ) and eMRCD 5 a (odds ratio 5.11) were the greatest predictors of inpatient mortality. The DECAF score has been further validated in external cohorts by subsequent studies, which have confirmed that DECAF is well-calibrated with excellent discrimination. $^{4,7,8}$

In 2018, a non-inferiority randomised controlled trial showed that low-risk (DECAF score 0-1) patients were suitable candidates for $\mathrm{HAH}$ treatment, with similar 90 -day readmission and mortality rates between $\mathrm{HAH}$ and usual care groups. This protocol comprised nebulisation, intravenous antibiotics, steroids, temporary oxygen at home as well as telephone or in-person review by a respiratory clinical nurse specialist, if necessary. ${ }^{5}$ At 14 days, $90 \%$ of patients across both arms stated that they would prefer HAH treatment for future exacerbations of comparable severity. The study also showed that mean 90-day costs were $£ 1,016$ lower in the HAH group, primarily due to reduced hospital admission days.

\section{Comparison to other prognostic tools}

Other scores have been produced to predict mortality in AECOPD patients. The BAP-65 metric is one such score, measuring serum urea, mental status, pulse and age. However, previous comparison of DECAF with BAP- 65 has highlighted the superiority of DECAF in predicting mortality. ${ }^{9}$ CURB- 65 has been shown to accurately predict mortality in community-acquired pneumonia, but its performance is relatively poor for AECOPD complicated by pneumonic consolidation. ${ }^{10,11}$ Accordingly, DECAF has been shown to be a better predictor of mortality in AECOPD patients than CURB-65. ${ }^{2}$ Indeed, its original external validation study showed that DECAF had significantly better discrimination for 30-day mortality than the CURB-65, CAPS, APACHE II and BAP- 65 scores. For inpatient mortality, DECAF also had significantly better 
discrimination compared to these scores, except for APACHE II where there was a non-significant difference. ${ }^{4}$

\section{Impact}

The 2017/18 COPD clinical audit showed a median inpatient stay of 4 days in AECOPD patients in the UK. ${ }^{2}$ Thus, our findings of mean stay for patients in PDSA cycle 1 were in keeping with the national average. We subsequently hope this QIP demonstrates a generalisable reduction in length of stay that could be achieved by other centres through adoption of a DECAF-based protocol for patients presenting with AECOPD. This reduction has the potential not only to benefit patient experience but also to decrease utilisation of hospital resources and reduce the financial burden on the NHS without compromising patient care. Following the success of this QIP, from 17 March 2020 onwards, the DECAF score has been approved for use in all AECOPD patients presenting to our centre with other centres in the local area looking to implement a similar protocol.

\section{Limitations}

Our project was limited by a low number of patients, and further planned data collection for PDSA cycle 2 was temporarily halted by the arrival of the global COVID-19 pandemic. However, all patients attending our centre in the specified data collection periods were assessed and included if eligible, and therefore we were still able to demonstrate the feasibility and safety of our improvements, albeit on a relatively small scale. Following resolution of the pandemic, further data collection with subsequent improvements is planned.

\section{Conclusion}

DECAF score can help clinicians in deciding the best possible care these patients receive, whether that is usual inpatient care or facilitated early discharge. This offers potential benefits to both the patient and hospital without worry of adverse events. The initial results of our QIP are limited by small patient cohorts and would benefit from further recruitment and assessment of outcomes. Nevertheless, we have successfully demonstrated the feasibility and safety of implementing a DECAF protocol at a busy DGH.

\section{Acknowledgements}

The authors acknowledge services of respiratory nurse specialists who played a massive role in implementation of DECAF score in Bedford Hospital.

\section{References}

1 NHS England. Overview of potential to reduce lives lost from Chronic Obstructive Pulmonary Disease (COPD). NHS, 2014. www.england.nhs.uk/wp-content/uploads/2014/02/rm-fs-6.pdf [Accessed 28 May 2020].

2 National Asthma and Chronic Obstructive Pulmonary Disease Audit Programme (NACAP). COPD clinical audit 2017/18. Royal College of Physicians, 2019. www.nacap.org.uk/nacap/welcome. nsf/vwFiles/COPD-2019-ClinicalAudit2017-18/\$File/COPD+Clinic al+Audit+2017_18+Audit+Report.pdf?openelement [Accessed 28 May 2020].

3 Steer J, Gibson J, Bourke S. The DECAF Score: predicting hospital mortality in exacerbations of chronic obstructive pulmonary disease. Thorax 2012;67:970-6.

4 Echevarria C, Steer J, Heslop-Marshall K et al. Validation of the DECAF score to predict hospital mortality in acute exacerbations of COPD. Thorax 2016;71:133-40.

5 Echevarria C, Gray ], Hartley T et al. Home treatment of COPD exacerbation selected by DECAF score: a non-inferiority, randomised controlled trial and economic evaluation. Thorax 2018;73: 713-22.

6 National Institute for Health and Care Excellence. Chronic obstruc tive pulmonary disease in over 16s: diagnosis and management: NICE guideline [NG115]. NICE, 2019. www.nice.org.uk/guidance/ ng115

7 Nafae R, Embarak S, Gad D. Value of the DECAF score in predicting hospital mortality in patients with acute exacerbation of chronic obstructive pulmonary disease admitted to Zagazig University Hospitals, Egypt. Egyptian Journal of Chest Diseases and Tuberculosis 2015;64:35-40.

8 Collier L, David T, Craig C, Yadavilli R. P45 practical use of the DECAF score: can we improve outcomes in acute exacerbation of COPD admissions? Thorax 2015;70(Suppl 3):A98.

9 Echevarria C, Steer J. The DECAF score is a superior predictor of in-hospital death than the BAP-65 score. J Clin Respir Dis Care 2016;2:1000111.

10 Lim WS, van der Eerden MM, Laing R et al. Defining community acquired pneumonia severity on presentation to hospital: an international derivation and validation study. Thorax 2003:58:377-82.

11 Steer J, Norman E, Afolabi O, Gibson G, Bourke S. Dyspnoea severity and pneumonia as predictors of in-hospital mortality and early readmission in acute exacerbations of COPD. Thorax 2012;67:117-21.

Address for correspondence: Dr Iftikhar Nadeem, Lister Hospital, Coreys Mill Lane, Stevenage SG1 4AB, UK. Email: iftikharnadeem49@gmail.com

Twitter: @iftikharnadeem8 